\title{
ISOTERMAS DE SORÇÃO DE FARINHA DE JABUTICABA: DETERMINAÇÃO EXPERIMENTAL PELO MÉTODO HIGROMÉTRICO E PREDIÇÃO TEÓRICA USANDO O SOFTWARE EMSO
}

\author{
C. L. LUCHESE, L. AMBROS, P. D. GURAK e L. D. F. MARCZAK \\ Universidade Federal do Rio Grande do Sul, Departamento de Engenharia Química \\ E-mail para contato: claudialuchese@yahoo.com.br
}

\begin{abstract}
RESUMO - Isotermas de sorção da farinha de jabuticaba foram determinadas experimentalmente em $10^{\circ} \mathrm{C}, 25^{\circ} \mathrm{C}$ e $40{ }^{\circ} \mathrm{C}$, através do método higroscópico, distinto do método padrão. As curvas apresentaram comportamento sigmoidal, característico de isotermas do Tipo II. Nas condições estudadas observou-se uma fraca influência da temperatura. Os modelos matemáticos de BET, GAB, Chirife, D’arcy Watt, Halsey, Oswin e Peleg se ajustaram bem aos dados experimentais sendo considerados satisfatórios. O modelo de Chirife apresentou o melhor ajuste dos dados experimentais $\left(\mathrm{R}^{2}>0,983\right)$. Adicionalmente, realizou-se a análise preditiva das isotermas através de uma metodologia recentemente disponibilizada na literatura, utilizando o software livre EMSO (Environment for Modeling, Simulation, and Optimization) para as temperaturas de $10{ }^{\circ} \mathrm{C}$ e $40{ }^{\circ} \mathrm{C}$. Para isso, somente os dados de atividade de água e umidade de equilíbrio da isoterma de $25{ }^{\circ} \mathrm{C}$ e o calor isostérico de sorção da amostra com o seu respectivo conteúdo de umidade foram utilizados. Observou-se um bom ajuste dos dados experimentais com as curvas preditas, com erro relativo médio inferior a $15 \%$.
\end{abstract}

\section{INTRODUÇÃO}

O processamento da jabuticaba para a produção de suco e derivados gera uma grande quantidade de bagaço, composto principalmente por sementes e cascas, representando em escala de laboratório aproximadamente $40 \%$ do peso total da fruta. Quando este bagaço é destinado para ração animal ou adubo seu uso é limitado devido à sua composição rica em fibras e compostos fenólicos. No entanto, são exatamente estas características que o tornam uma matéria-prima de interesse para o seu reaproveitamento pela indústria de alimentos (Leite et al., 2011). Desta forma, agregar valor a este subproduto é de interesse econômico e ambiental, necessitando de investigação científica e tecnológica para que sua utilização possa ser realizada de forma eficiente, econômica e segura, por exemplo, na produção de farinha de jabuticaba.

Sendo assim, a determinação das isotermas de sorção dessa farinha em diferentes temperaturas faz-se necessária, uma vez que a análise destas curvas propicia informações para o planejamento de 
processos, como a umidade ótima para a estabilidade de um produto desidratado em relação à oxidação de lipídios, atividade enzimática, preservação de componentes do sabor e características estruturais. Permitindo aliar a segurança microbiológica e viabilidade econômica durante o processo de secagem.

Dentro deste contexto, o presente trabalho objetivou determinar experimentalmente as isotermas de sorção de farinha de jabuticaba em diferentes temperaturas $\left(10{ }^{\circ} \mathrm{C}, 25^{\circ} \mathrm{C}\right.$ e $\left.40{ }^{\circ} \mathrm{C}\right)$ através do método direto higroscópico. Essa metodologia é distinta do método padrão comumente apresentado na literatura, que possui como inconveniente o longo tempo de espera necessário para a obtenção dos dados experimentais, uma vez que as amostras demoram a entrar em equilíbrio com as atmosferas salinas a que são submetidas. Adicionalmente foram realizadas: (i) a modelagem matemática das curvas a fim de avaliar os melhores ajustes aos resultados experimentais, e (ii) a análise preditiva das isotermas através de uma metodologia recentemente disponibilizada na literatura por Staudt et al. (2013) utilizando o software livre EMSO (Environment for Modeling, Simulation, and Optimization).

\section{MATERIAIS E MÉTODOS}

\subsection{Matéria-prima}

A farinha de jabuticaba utilizada neste experimento foi produzida utilizando o bagaço gerado no processo de prensagem na produção de suco integral. Posteriormente este bagaço foi seco com circulação de ar $\left(60^{\circ} \mathrm{C}\right.$ por 72 horas), triturado e peneirado (malha de 32 mesh) para a obtenção da farinha de jabuticaba.

\subsection{Construção das isotermas de sorção}

Amostras da farinha de jabuticaba foram colocadas em contato com diferentes atmosferas de umidade relativa de $90 \%$ (solução saturada de sulfato de cobre), e de $10 \%$ (solução saturada de hidróxido de potássio), em diferentes tempos, para obtenção de amostras com diferentes teores de atividade de água. $\mathrm{O}$ conteúdo de umidade foi determinado pelo método gravimétrico de acordo com a metodologia 925.10 (AOAC, 2005). Os valores experimentais de atividade de água $\left(\mathrm{a}_{\mathrm{w}}\right)$ foram obtidos através de leitura direta em triplicata em medidor eletrônico (Novasina, modelo Labmaster), setando-se cada uma das temperaturas de análise.

\subsection{Modelagem Matemática de Isotermas de Sorção}

Os dados de sorção obtidos experimentalmente foram ajustados aos modelos matemáticos apresentados na Tabela 1 utilizando o módulo de análises de regressão não linear do programa Statistica 8.0 (Statsoft, USA, 1998), onde $X_{\mathrm{m}}$ e $\mathrm{X}_{\mathrm{eq}}$ são, respectivamente, os teores de umidade de equilíbrio e de monocamada ( $\mathrm{g}$ água $\mathrm{g}$ amostra seca ${ }^{-1}$ ); $\mathrm{a}_{\mathrm{w}}$ é a atividade de água ( $\mathrm{g}$ água $\mathrm{g}$ ar $\mathrm{seco}^{-1}$ ) enquanto que $\mathrm{A}, \mathrm{B}, \mathrm{C}, \mathrm{K}, \mathrm{K}_{1}, \mathrm{~K}_{2}, \mathrm{~K}_{3}, \mathrm{~K}_{4}, \mathrm{~K}_{5}, \mathrm{n}_{1}$ e $\mathrm{n}_{2}$ são as constantes de cada modelo. 
Tabela 1 - Modelos matemáticos utilizados para ajuste de isotermas de sorção.

\begin{tabular}{|c|c|}
\hline Modelo & Equação \\
\hline BET (Brunauer, Emmet e Teller, 1938) & $\frac{a_{w}}{\left(1-a_{1 W}\right) \cdot X_{E q}}=\frac{1}{X_{m} C_{B E T}}+\frac{a_{w w}\left(C_{B E T}-1\right)}{X_{m m^{N}} C_{B E T}}$ \\
\hline GAB (Van den Berg, 1985) & $X_{e q}=\frac{\left(X_{m} C_{x} K_{x} a_{W}\right)}{\left(1-K_{x} a_{w}\right) \times\left(1-K_{x} a_{w}+C_{x} K_{x} a_{w}\right)}$ \\
\hline Chirife (Castillo et al., 2008) & $X_{e q}=\exp \left[A+B \cdot \ln \left(C-\ln a_{w n}\right)\right]$ \\
\hline D'arcy Watt (D'arcy e Watt, 1970) & $X_{\varepsilon q}=\frac{K_{1}, K_{2}, a_{w}}{1+K_{1}, a_{w}}+K_{5}, a_{w}+\frac{K_{\mathrm{a}}, K_{4} \times a_{w}}{1-K_{\mathrm{a}} \times a_{w w}}$ \\
\hline Halsey (Halsey, 1948) & $\ln \left(a_{w q}\right)=\left(\frac{-A}{X_{e q}^{B}}\right)$ \\
\hline Oswin (Lomauro, Bakshi e Labuza, 1985) & $X_{e q}=A_{\cdot}\left(\frac{a_{w}}{1-a_{w}}\right)^{B}$ \\
\hline Peleg (Peleg, 1993) & $X_{e q}=K_{1} \times a_{W}^{n 1}+K_{2} \times a_{W}^{n 2}$ \\
\hline
\end{tabular}

Para a avaliação da acurácia do ajuste de cada modelo foram utilizados o coeficiente de determinação $\left(\mathrm{R}^{2}\right)$ e o erro relativo médio (ERM), de acordo com a equação (1):

$\operatorname{ERM}(\%)=\frac{100}{N} \sum_{\mathrm{i}=1}^{N} \frac{\left|a_{e \mathrm{i}}-a_{\mathrm{pi}}\right|}{a_{e \mathrm{i}}}$

onde $a_{e}$ e $a_{p}$ são, respectivamente, os valores de umidade de equilíbrio experimentais e preditos pelos modelos ( $\mathrm{g}$ água $\mathrm{g}$ sólidos $\operatorname{secos}^{-1}$ ) e $\mathrm{N}$ representa o número de pontos experimentais. Geralmente é considerado que valores de ERM menores do que $10 \%$ indicam um ajuste adequado do modelo matemático (Lomauro, Bakshi e Labuza, 1985).

\subsection{Análise preditiva usando o software EMSO}

Realizou-se a análise preditiva das isotermas utilizando o modelo matemático de GAB juntamente com a equação de Clausius-Clapeyron utilizando um simulador de processos, o software livre EMSO (Environment for Modeling, Simulation, and Optimization) para as temperaturas de $10{ }^{\circ} \mathrm{C}$ e $40{ }^{\circ} \mathrm{C}$. Para isso, somente os dados de atividade de água e umidade de equilíbrio da isoterma de $25^{\circ} \mathrm{C}$ e o calor isostérico de sorção da amostra com o seu respectivo conteúdo de umidade foram utilizados, de acordo com a metodologia proposta por Staudt et al. (2013).

\section{RESULTADOS E DISCUSSÃO}

\subsection{Determinação experimental de isotermas de sorção}

A Figura 1 apresenta as isotermas de sorção de farinha de jabuticaba determinadas experimentalmente nas três temperaturas estudadas. Todos os resultados experimentais obtidos 


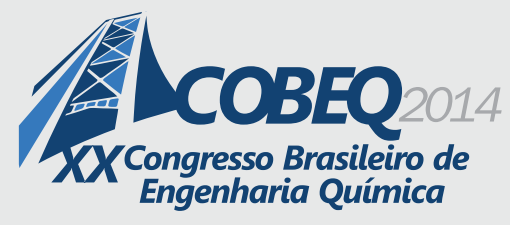

19 a 22 de outubro de 2014
Florianópolis/SC

apresentaram um coeficiente de variação inferior a $4 \%$ para os valores de atividade de água, e inferior a $6 \%$ para os valores de umidade. É possível verificar que para uma dada temperatura, o teor de umidade do produto aumenta com a atividade de água. Este comportamento era esperado, pois a pressão de vapor de água presente no produto acompanha o aumento da pressão de vapor do meio que o envolve. Também foi observado um aumento repentino da umidade de equilíbrio para o último ponto observado no gráfico, comportamento típico de produtos com elevada higroscopicidade (Medeiros et al., 2006).

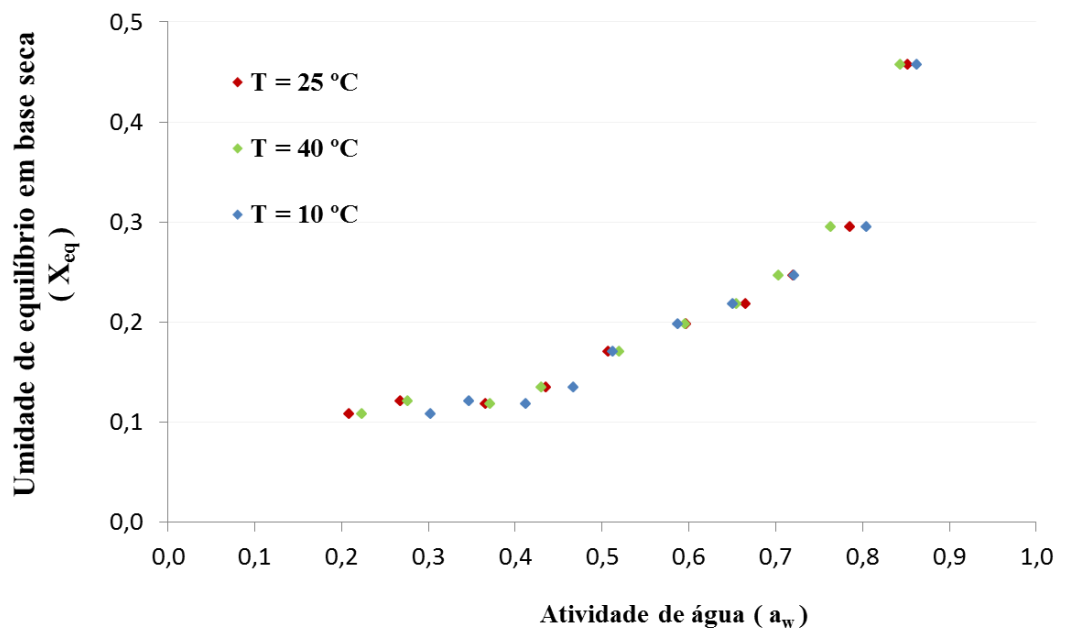

Figura 1 - Isotermas de sorção de farinha de jabuticaba determinadas experimentalmente nas temperaturas de $10^{\circ} \mathrm{C}, 25^{\circ} \mathrm{C}$ e $40^{\circ} \mathrm{C}$. Todos os dados experimentais apresentaram coeficiente de variação inferior a $6 \%$.

Adicionalmente verificou-se que as três isotermas para a farinha de jabuticaba nem sempre apresentaram uma separação consistente ao longo de toda a curva, apresentando até mesmo, alguns pontos sobrepostos, indicando fraca influência da temperatura nesta propriedade. Um comportamento similar foi observado em proteína de soja texturizada com isotermas realizadas nas temperaturas de 10 a $40{ }^{\circ} \mathrm{C}$, em grãos de cacau não fermentados, para as temperaturas de 25 a $40{ }^{\circ} \mathrm{C}$ e em farinha de pinhão, para as temperaturas de 10 a $40^{\circ} \mathrm{C}$ (Cassini, Marczak e Noreña, 2006; Cladera-Olivera et al., 2011). Como a farinha de jabuticaba apresenta em sua composição amido e proteínas, esses componentes podem afetar os dados de sorção, pois são macromoléculas ricas em grupos polares que se comportam como centros ativos de sorção, podendo ocasionar maior influência no comportamento das isotermas do que na variação da temperatura (Erbaş, Ertugay e Certel, 2005).

\subsection{Avaliação de modelos matemáticos}

A Tabela 2 apresenta os parâmetros matemáticos calculados a partir das equações apresentadas na Tabela 1, assim como o coeficiente de correlação $\left(\mathrm{R}^{2}\right)$ e o erro relativo médio (ERM) para cada temperatura. Os modelos de BET, GAB, Chirife, D’arcy Watt, Halsey, Oswin e Peleg foram considerados satisfatórios, por apresentarem coeficiente de determinação próximos da unidade e erro relativo médio menor do que 10\%. O modelo de Chirife apresentou o melhor ajuste aos dados experimentais nas três temperaturas. 
Tabela 2 - Valores dos parâmetros matemáticos de ajuste calculados para as isotermas determinados experimentalmente.

\begin{tabular}{|c|c|c|c|c|c|}
\hline \multirow{2}{*}{ Modelo } & \multirow{2}{*}{ Constante } & \multicolumn{3}{|c|}{ Temperaturas } & \multirow{2}{*}{ Média } \\
\hline & & $10^{\circ} \mathrm{C}$ & $25^{\circ} \mathrm{C}$ & $40^{\circ} \mathrm{C}$ & \\
\hline \multirow{4}{*}{ BET } & $X_{m}$ & 0,069 & 0,071 & 0,075 & \\
\hline & $\mathrm{C}$ & 1800448 & 6792369 & 9126679 & \\
\hline & $\mathrm{R}^{2}$ & 0,900 & 0,954 & 0,978 & \\
\hline & ERM (\%) & 10,87 & 10,98 & 6,44 & 9,43 \\
\hline \multirow{5}{*}{ GAB } & $\mathrm{X}_{\mathrm{m}}$ & 0,062 & 0,079 & 0,079 & \\
\hline & $\mathrm{C}$ & 167971405 & 82382 & 21570 & \\
\hline & $\mathrm{K}$ & 0,717 & 0,962 & 0,977 & \\
\hline & $\mathrm{R}^{2}$ & 0,954 & 0,981 & 0,992 & \\
\hline & ERM (\%) & 7,42 & 5,95 & 4,47 & 5,95 \\
\hline \multirow{5}{*}{ Chirife } & $\mathrm{A}$ & $-2,10$ & $-2,07$ & $-2,08$ & \\
\hline & $\mathrm{B}$ & $-0,46$ & $-0,44$ & $-0,53$ & \\
\hline & $\mathrm{C}$ & $-0,09$ & $-0,11$ & $-0,07$ & \\
\hline & $\mathrm{R}^{2}$ & 0,98 & 0,99 & 0,99 & \\
\hline & ERM (\%) & 7,09 & 4,15 & 3,39 & 4,87 \\
\hline \multirow{7}{*}{ D'arcy e Watt } & $\mathrm{K}_{1}$ & $-1,12$ & $-0,41$ & $-0,49$ & \\
\hline & $\mathrm{K}_{2}$ & 0,35 & 2,06 & 2,70 & \\
\hline & $\mathrm{K}_{3}$ & 1,12 & 1,02 & 0,89 & \\
\hline & $\mathrm{K}_{4}$ & 0,35 & 0,08 & 0,38 & \\
\hline & $\mathrm{K}_{5}$ & 0,32 & 1,25 & 1,50 & \\
\hline & $\mathrm{R}^{2}$ & 0,99 & 0,99 & 0,99 & \\
\hline & ERM (\%) & 4,99 & 7,01 & 5,81 & 5,94 \\
\hline \multirow{4}{*}{ Halsey } & $\mathrm{A}$ & 0,05 & 0,03 & 0,03 & \\
\hline & $\mathrm{B}$ & 1,43 & 1,81 & 1,70 & \\
\hline & $\mathrm{R}^{2}$ & 0,96 & 0,95 & 0,95 & \\
\hline & ERM (\%) & 9,27 & 10,65 & 9,61 & $\mathbf{9 , 8 4}$ \\
\hline \multirow{4}{*}{ Oswin } & $\mathrm{A}$ & 0,15 & 0,16 & 0,16 & \\
\hline & $\mathrm{B}$ & 0,56 & 0,54 & 0,58 & \\
\hline & $\mathrm{R}^{2}$ & 0,96 & 0,95 & 0,96 & \\
\hline & ERM (\%) & $\mathbf{7 , 4 2}$ & 9,72 & 8,68 & 8,61 \\
\hline \multirow{6}{*}{ Peleg } & $\mathrm{k}_{1}$ & 11,31 & 0,26 & 0,24 & \\
\hline & $\mathrm{n}_{1}$ & 28,41 & 0,65 & 0,58 & \\
\hline & $\mathrm{k}_{2}$ & 0,34 & 1,60 & 1,08 & \\
\hline & $\mathrm{n}_{2}$ & 1,07 & 12,43 & 8,85 & \\
\hline & $\mathrm{R}^{2}$ & 0,99 & 0,99 & 0,99 & \\
\hline & ERM (\%) & 5,07 & 6,28 & 5,08 & 5,48 \\
\hline
\end{tabular}




\section{9 a 22 de outubro de 2014 \\ Florianópolis/SC}

Os valores de umidade de monocamada $\left(\mathrm{X}_{\mathrm{m}}\right)$ obtidos a $25^{\circ} \mathrm{C}$ foram 0,071 (g água.g sólidos $\operatorname{secos}^{-1}$ ) pelo modelo de BET e 0,079 (g água.g sólidos secos ${ }^{-1}$ ) pelo modelo de GAB. Estes valores são compatíveis com os encontrados por Chirife e Iglesias (1978) e Yanniotis (1994), que reportaram teores de umidade de monocamada médios de 0,0736 (g água.g sólidos ${ }^{-1}$ ) para alimentos ricos em carboidratos na temperatura de $30^{\circ} \mathrm{C}$ através do modelo de BET. Este parâmetro é importante para predizer as condições de armazenamento e deterioração de alimentos, uma vez que indica a quantidade de água fortemente adsorvida aos sítios específicos na superfície do produto, fornecendo o conteúdo de água no qual se tem a estabilidade máxima de um produto desidratado. Em valores de umidade abaixo da umidade de monocamada, a taxa das reações de deterioração, exceto a oxidação de lipídios, é mínima (Goula et al., 2008).

Analisando a Figura 1 e o resultado dos parâmetros do modelo de GAB é possível comprovar que as isotermas encontradas correspondem ao Tipo II, sigmoidal, de acordo com a classificação de $\mathrm{BET}$, visto que os valores da constante $\mathrm{C}$ foram maiores do que 10 e que os valores de $\mathrm{K}$ permaneceram na faixa entre 0 e 1 (Blahovec, 2004). Aguirre-Cruz et al. (2010), Cladera-Olivera et al. (2011), Chisté et al. (2012) e Spada et al. (2013) também reportaram comportamentos similares para isotermas de farinha de banana, farinha de pinhão, farinha de tapioca e amido de pinhão, respectivamente.

\subsection{Determinação teórica do calor isostérico de sorção}

O calor isostérico de sorção $\Delta \mathrm{h}\left(\mathrm{J}_{\mathrm{m}} \mathrm{mol}^{-1}\right)$ foi determinado através da equação de Clausius Clapeyron linearizada, através da análise de regressão linear, e pode ser visualizado na Figura 2.

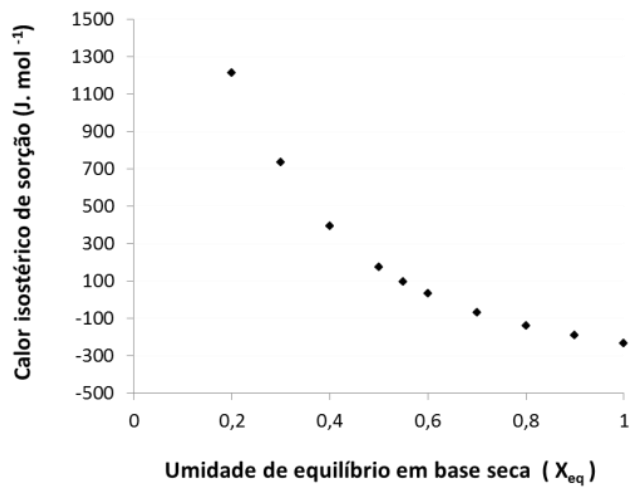

Figura 2 - Calor isostérico de sorção de farinha de jabuticaba.

O comportamento da curva foi compatível com o esperado: quanto maior o teor de umidade das amostras, maiores serão os valores de calor isostérico de sorção. Varghese et al. (2012), Spada et al, . (2013) e Majd et al., (2013) reportaram curvas similares para suco de uva em pó, amido de pinhão e sementes de uva, respectivamente. Esse comportamento é explicado por Shivhare et al. (2004), visto que em baixos teores de umidade ocorre uma forte interação entre o produto e a água presente nele, sendo necessário elevado valor de energia para retirar a água contida na amostra. Esta propriedade pode ser utilizada para estimar o requerimento de energia do processo de desidratação do alimento até seu valor de umidade da monocamada ou para obtenção de uma determinada umidade final desejada. 


\subsection{Predição teórica de isotermas de sorção em diferentes temperaturas}

A isoterma utilizada como base de cálculo foi àquela determinada na temperatura de $25^{\circ} \mathrm{C}$. $\mathrm{O}$ calor isostérico de $4520 \mathrm{~J} . \mathrm{mol}^{-1}$ para uma amostra de farinha de jabuticaba com umidade de $0,155 \mathrm{~g}$ água.g sólidos $\operatorname{secos}^{-1}$. É possível verificar, através da Figura 3 , que foi obtida uma boa correlação das curvas preditas com os dados experimentais, com erro relativo médio de 11,4\% e $15,0 \%$, para as temperaturas de $10^{\circ} \mathrm{C}$ e $40^{\circ} \mathrm{C}$, respectivamente. Esta mesma comparação foi realizada por Staudt et al. (2013) para o ajuste de isotermas de sorção de couve-flor, apresentando erro relativo médio de $11,5 \%$, e para polpa de tomate, com erro relativo médio de $8,5 \%$.
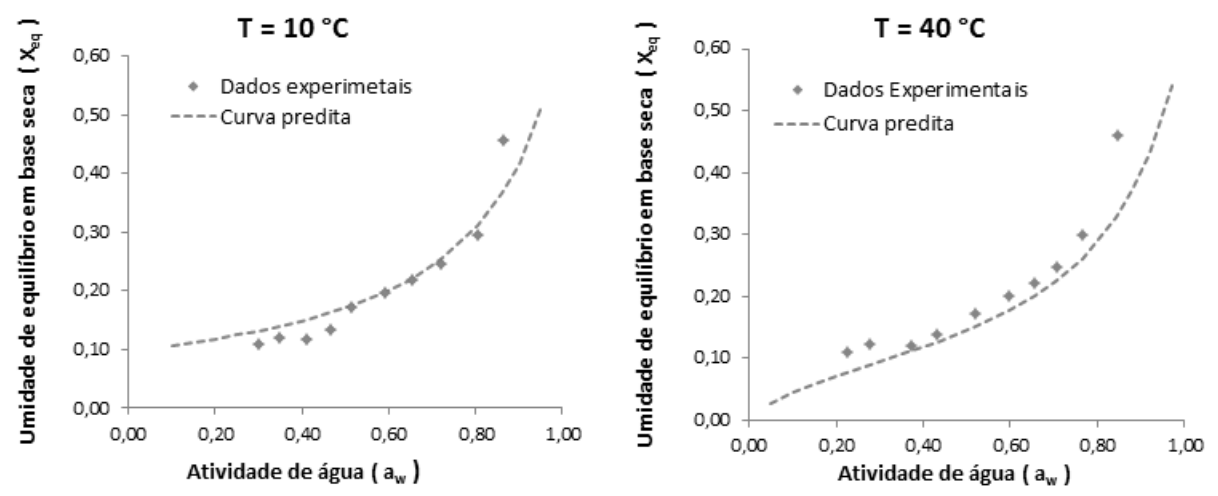

Figura 3 - Ajuste dos dados experimentais para determinação das isotermas de farinha de jabuticaba nas temperaturas de $10^{\circ} \mathrm{C}$ e $40^{\circ} \mathrm{C}$ usando uma nova metodologia de predição.

\section{REFERÊNCIAS}

AGUIRRE-CRUZ, A.; CARMONA-GARCÍA, R.; BELLO-PÉREZ. Moisture adsorption behavior of banana flours (Musa paradisiaca) um modified and modified by acid-treatment. Starch, v. 62, p. 658-666, 2010.

AOAC. Official methods of analysis. 8 ed., Association of Official Analytical Chemists, Washington, DC, 2005.

BLAHOVEC, J. Sorption isotherms in materials of biological origin mathematical and physical approach. $J$. Food Eng., v. 65, n. 4, p. 489-495, 2004.

BRUNAUER, S.; EMMETT, P. H.; TELLER, E. Adsorption of gases in multi molecular layers. J. Am. Chem. Soc., v. 60, p. 309-319, 1938.

CASSINI, A. S.; MARCZAK, L. D. F.; NOREÑA, C. P. Z. Water adsorption isotherms of texturized soy protein. J. Food Eng., v. 77, p. 194-199, 2006.

CASTILlo, M.D.; MARÍNEZ, E. J.; GONZÁlEZ, H. H. L.; PACIN, A. M.; RESNIK, S. L. Study of mathematical models applied to sorption isotherms of Argentinean black bean varieties. J. Food Eng., v. 60, p. 343-348, 2008.

CHIRIFE, J.; IGLESIAS H. A. Equations for fitting water sorption isotherms of foods. Part I - A review. Int. J. Food Sci. Tech., v. 13, p. 159-174, 1978. 
CHISTÉ, R. C.; SILVA, P. A.; LOPES, A. S.; PENA, R. S. Sorption isotherms of tapioca flour. Int. J. Food Sci. Tech., v. 47, p. 870-874, 2012.

CLADERA-OLIVERA, F.; MARCZAK, L. D. F.; NOREÑA, C. P. Z.; PETTERMANN, A. C. Modeling water adsorption isotherms of pinhão (Araucaria angustifolia seeds) flour and thermodynamic analysis of the adsorption process. J. Food Process Eng., v. 34, p. 826-843, 2011.

D'ARCY, R. L; WATT, I. C. Analysis of sorption isotherms of nonhomogeneous sorbents. Transaction Faraday Society, v. 66, p. 1236-1240, 1970.

ERBAŞ, M.; ERTUGAY, M. F.; CERTEL, M. Moisture adsorption behavior of semolina and farina. J. Food Eng., v. 69, p. 191-198, 2005.

GOULA, A. M.; KARAPANTSIOS, T. D.; ASCHILIAS, D. S.; ADAMOPOULOS, K. G. Water sorption isotherms and glass transition temperature of spray dried tomato pulp. J. Food Eng., v. 85, p. 73-83, 2008.

HALSEY, G. Physical desorption on non-uniform surfaces. J. Chem. Phys., v. 16, p. 931-937, 1948.

LEITE, A. V; MALTA, L. G.; RICCIO, M. F.; EBERLIN, M. N.; PASTORE, G. M.; MARÓSTICA JÚNIOR, M. R. Antioxidant potential of rat plasma by administration of freeze-dried jaboticaba peel (Myrciaria jaboticaba Vell Berg). J. Agr. Food Chem., vol. 59, p. 2277-2283, 2011.

LOMAURO, C. J.; BAKSHI, A. S.; LABUZA, T. P. Evaluation of food moisture sorption isotherm equations part I: Fruit, vegetable and meat products. $L W T$, v. 18, p. 111-117, 1985.

MAJD, K. M.; KARPARVARFARD, S. H.; FARAHNAKY, A; JAFARPOUR, K. Thermodynamic of water sorption of grape seed: temperature effect of sorption isotherms and thermodynamic characteristics. Food Biophysics, v. 8, p. 1-11, 2013.

MEDEIROS, M. L.; AYROSA, A. M. I. B.; PITOMBO, R, N. M.; LANNES, S. C. S. Sorption isotherms of cocoa and cupuassu products. J. Food Eng., v. 73, p. 402-406, 2006.

PELEG, M. Assessment of a semi empirical four parameter general model for sigmoid moisture sorption isotherms. J. Food Process Eng., v. 16, p. 21-37, 1993.

SHIVHARE, U. S.; ARORA, S.; AHMED, J.; RAGHAVAN, G. S. V. Moisture adsorption isotherms for mushroom. LWT, v. 37, p. 133-137, 2004.

SPADA, J. C.; NOREÑA, C. P. Z.; MARCZAK, L D. F.; TESSARO, I. C. Water adsorption isotherms of microcapsules with hydrolyzed pinhão (Araucaria angustifolia seeds) starch as wall material. J. Food Eng., v. 114, p. 64-69, 2013.

STAUDT, P. B.; TESSARO, I. C.; MARCZAK, L. D. F., SOARES, R. de P., CARDOZO, N. S. M. A new method for predicting sorption isotherms at different temperatures: Extension to the GAB model. J. Food Eng., v. 118, p. 247-255, 2013.

VAN DEN BERG, C. Development of B.E.T. like models for sorption of water of foods, theory and relevance. Properties of water in foods, Martinus Nijhoft, Dordrecht, p.119-135, 1985.

VARGHESE, K. S.; RADHAKRISHNA, K.; BAWA, A. S. Moisture sorption characteristics of freeze dried whey-grape beverage mix. J. Food Sci. Tech., p. 1-7, 2012.

YANNIOTIS, S. A new method for interpolating and extrapolating water activity data. J. Food Eng., v. 21, p. 81-96, 1994. 